\title{
EuroBloodNet: From Rare Anaemias to Rare Haematological Diseases, a Transitional Proposal for European Reference Network (ERN)
}

\author{
Joan Lluis Vives Corrons
}

Potential Coordinator of EuroBloodNet, Red Cell Pathology Unit, Hospital Clínic, Barcelona, Spain

Corresponding author: Joan Lluis Vives Corrons, Professor, Red Cell Pathology Unit, Hospital Clínic, Block 9, Floor 0, Villarroel 170 Street, Barcelona 08036, Spain, Tel: +34934515950, Fax: +34932271764; E-mail: JLVIVES@clinic.cat

Received date: July 03, 2016; Accepted date: July 07, 2016; Published date: July 14, 2016

Copyright: (C) 2016 Vives Corrons JL. This is an open-access article distributed under the terms of the Creative Commons Attribution License, which permits unrestricted use, distribution and reproduction in any medium, provided the original author and source are credited.

Citation: Vives Corrons JL. Eurobloodnet: From Rare Anaemias to Rare Haematological Diseases, a Transitional Proposal for European Reference Network. J Rare Dis Diagn Ther. 2016, 2:4.

\section{Editorial}

Rare diseases (RD) are conditions that affect less than 5 per 10,000 individuals in the general population, and that require a concentration of resources and expertise in order to provide high-quality, cost-effective care. Since 2002, the European Reference Network for rare and Congenital Anaemias (ENERCA) has contributed to promote two pivotal aspects of the 2011 EU Directive on patients' rights in cross-border healthcare: 1) A specific framework for cross-border healthcare and 2) A European cooperation for helping in the diagnosis and follow up of patients [1]. After 2013, the EC has approved the co-financing of ENERCA Project for a further three years (2014-2016), with the aim of implementing ehealth information and communication technologies (ICT) in order to assure the same access to health services, independently from the place of residence. This new Project, called e-ENERCA, is using the most innovative ICT to create a pan-European interoperable e-health platform for electronic registry (e-registry), on-line teaching (e-learning) and telediagnosis (e-medicine), avoiding, when possible, the need of patient's or experts travelling.

Article 12 of the 2011 EU Directive mentioned before, sets out a mix of Commission responsibilities and provisions to support Member States (MS) in the development of European Reference Networks (ERN) linking healthcare providers (HCP) and centres of expertise (CoE) from the different EU Member States [2]. However, since there are more than 7,000 Rare Diseases (RD), in order to avoid individual RD applications for ERN, the EC has grouped all these diseases into 23 different ERN, essentially based of medical specialties (Table 1). Based on more than 12 years of experience in networking and RAs healthcare recommendations [3], ENERCA has decided to apply for ERN in Rare haematological diseases (RHD) by expanding its working plan to white blood cell and platelets disorders, in addition to rare anaemias. After the publication of the Call for Interest for ERN on March 2016 [4], ENERCA and the European Haematology Association (EHA) have decided to prepare a common application for a ERN in Rare Haematological Diseases, known as EuroBloodNet, that includes non-oncological or benign" (Table 2) and oncological or "malignant" (Table 3) RHD. According to EC requirements for ERN application, it is mandatory to include a minimum of 10 Health Providers (HCP) from at least 8 Member States (MS).

These objectives are largely accomplished by EuroBloodNet that, if approved, will implement 5 main coordinator actions: 1) Enhance the equality in the care delivery for RHD across Europe by the establishment of and European inventory of experts, centres, and facilities in RHD, Mapping of European centres of Expertise (CoE), collecting patient's data and patient's registry and assure the cross border patient's transferal system. 2) Facilitate the harmonization of the haematology specialties curricula by improving continuing medical education on RHD (Training courses), promoting blended learning by on site and on-line learning (e-learning platform) and promote the diffusion and implementation of existing guidelines, 3) Provide interprofessional consultation by sharing of knowledge and exchange of clinical information. This will contribute to the decrease in the number of undiagnosed/misdiagnosed patients, facilitate the remote diagnosis of complex cases (Telemedicine platform) and improve patient care by building bridges between health professionals and experts in distant locations, 4) Promote the best practices in prevention, diagnosis and clinical care across Europe by gathering of existing protocols, national programs, recommendations and guidelines, promoting harmonization of procedures for diagnosis and the development of EQAS, assessment of existing guidelines and/or implementation and developing new guidelines and, 5) Foster European cooperation in specialized diagnosis, health care and research by promoting clinical trials, facilitate the provision of omics platforms and new technologies and provide support research in drug-able targets identification, gene therapy and pathophysiology.

To implement these different actions, EuroBloodNet will use the same transversal fields of support action as used by ENERCA, but expanded to all rare haematological diseases: Registry, Training, Telemedicine, Guidelines \& Prevention and research \& Clinical trials [5]. 
Table 1: Proposed European Reference Networks (ERNs).

\begin{tabular}{|c|c|}
\hline $\begin{array}{l}\text { SI } \\
\text { No. }\end{array}$ & Proposed European Reference Networks (ERNs) \\
\hline 1 & Rare auto-immune and auto inflammatory diseases \\
\hline 2 & Rare bone diseases \\
\hline 3 & Rare cancers and tumours paediatric \\
\hline 4 & Rare cancers and tumours adults \\
\hline 5 & Rare cardiac diseases \\
\hline 6 & Rare connective tissue and musculoskeletal diseases \\
\hline 7 & $\begin{array}{l}\text { Rare craniofacial anomalies and ENT (ear, nose and throat) } \\
\text { disorders }\end{array}$ \\
\hline 8 & Rare endocrine diseases \\
\hline 9 & Rare eye diseases \\
\hline 10 & Rare gynaecological and obstetric diseases \\
\hline 11 & Rare haematological diseases \\
\hline 12 & Rare liver diseases \\
\hline 13 & Rare inherited metabolic disorders \\
\hline 14 & $\begin{array}{l}\text { Rare malformations, developmental anomalies and intellectual } \\
\text { disabilities }\end{array}$ \\
\hline 15 & Rare gastrointestinal diseases \\
\hline 16 & Rare neurological diseases \\
\hline 17 & Rare neuromuscular diseases \\
\hline 18 & Rare pulmonary diseases \\
\hline 19 & Rare renal diseases \\
\hline 20 & Rare skin disorders \\
\hline 21 & Rare urogenital diseases \\
\hline 22 & Rare Vascular diseases \\
\hline 23 & Transplantation in children \\
\hline
\end{tabular}

Table 2: Eurobloodnet: Rare non-oncological diseases.

Eurobloodnet: Rare non-oncological diseases

\section{1) Rare red blood Cell (RBC) defects}

Haemoglobinopathies (ORPHA 68364)

Thalassemias (Intermedia and Major)

Sickle-cell disease (SCD) and other structural haemoglobinopathies

Unstable haemoglobinopathies

Congenital methaemoglobinaemia due to $\mathrm{HbM}$

\section{Rare haemolytic anaemias (ORPHA 98363)}

Hereditary erythroenzymopathies (ORPHA 98369)

Hereditary RBC membrane defects (ORPHA 98364)

Acquired haemolytic anaemias and related disorders ( ORPHA 182047)

\section{Rare congenital erythrocytosis}

Primary congenital erythrocytosis due abnormal hypoxia sensing (ORPHA 90042)

Haemoglobinopathies with high affinity for oxygen (ORPHA 68364)
Erythrocyte biphosphoglycerate mutase (BPGM) deficiency

Familial neonatal cyanosis

Cytochrome b5 reductase (diaphorase) deficiency

Haemoglobinopathies with low affinity for oxygen (ORPHA 68364)

2) Bone marrow (BM) failure and rare haematopoietic disorders

Bone marrow failure (BMF)

Aplastic anaemia (ORPHA 182040)

Fanconi anaemina (ORPHA 84)

Paroxysmal nocturnal haemoglobinuria PNH (ORPHA 447)

Rare haematopoietic disorders

Blackfan-diamond anaemia BDA (ORPHA 124)

Congenital dyserythropoietic anemias, CDA (ORPHA 85)

Congenital neutropaemia and related disorders (ORPHA 42738)

Histiocytosis and related disorders

3) Rare bleeding/coagulation disorders and related diseases

Rare thrombotic disease of hematologic origin (ORPHA 182054)

Thrombotic thrombocytopenic purpura (ORPHA 54057)

Atypical hemolytic-uremic syndrome (ORPHA 2134)

Familial thrombocytosis (ORPHA 71493)

\section{Rare hemorrhagic disorder (ORPHA 248308)}

Hemophilia A (Factor VIII deficiency) (ORPHA 98878)

Hemophilia B (Factor IX deficiency) (ORPHA 98879)

Von willebrand disease (ORPHA 903)

Congenital fibrinogen (Factor I) deficiency (ORPHA 335)

Congenital prothrombin (Factor II) deficiency (ORPHA 325)

Congenital factor VII deficiency (ORPHA 327)

Congenital factor XII deficiency (ORPHA 330)

Congenital Factor XIII deficiency (ORPHA 331)

Immune thrombocytopaenic purpura (ITP)

Inherited platelet disorders

Hereditary thrombophilia

Familial dysfibrinogenemia (ORPHA 98881)

Protein $\mathrm{C}$ deficiency (ORPHA 745)

Protein S deficiency (ORPHA 743)

Hyperhomocysteinaemia/homocysteinuria (ORPHA 394)

4) Haemochromatosis and rare hereditary iron metabolism disorders

Rare hemochromatosis

type 2 (ORPHA 79230), type 3 (ORPHA 225123), type 4 (ORPHA 139491)

Rare anaemia due to iron metabolism defects (ORPHA 98360)

X-linked sideroblastic anaemia (ORPHA 1047)

Erythropoietic porphyria (ORPHA 79277)

Table 3: Eurobloodnet: Rare oncological diseases.

Eurobloodnet: Rare oncological diseases

1) Myeloid malignancies (ORPHA 171895)

Acute myeloid leukemia, AML (ORPHA 519)

Myelodysplastic syndromes, MDS (ORPHA 52688)

Chronic myeloproliferative neoplasms (ORPHA 98274)

Chronic myeloid leukemia (ORPHA 521)

Polycythemia vera (ORPHA 729)

Essential thrombocythemia (ORPHA 3318)

Idiopathic myelofibrosis 
Hypereosinophilic syndrome (ORPHA 168956)

Systemic mastocytosis (ORPHA 2467)

\section{2) Lymphoid malignancies (ORPHA 171898)}

Acute lymphoblastic leukemia (ORPHA 513)

Chronic lymphoproliferative disorders

B-cell/T-cell prolymphocytic leukemia (ORPHA 86852)

Hairy cell leukemia (ORPHA 58017)

T-cell large granular lymphocytic leukaemia (ORPHA 86872) and other atypical CLL

Mycosis fungoides, Sezary syndrome and variants (ORPHA 178566) Lymphoma (ORPHA 223735) including

Follicular lymphoma (ORPHA 545)

Mantle cell lymphoma (ORPHA 52416)

Burkitt lymphoma (ORPHA 543)

Marginal zone lymphoma (ORPHA 300912)

Immunodeficiency-associated lymphoproliferative disease (ORPHA 98290)

Post-transplant lymphoproliferative disease (ORPHA 70568)

Primary cutaneous lymphoma (ORPHA 542)

EBV-positive diffuse large B-cell lymphoma of the elderly (ORPHA 289661)

Adult T-cell leukemia/lymphoma (ORPHA 86875)

Primary central nervous system (PCNS) lymphoma (ORPHA 46135)

Monoclonal gammopathies

Specific aspects of myeloma (not typical myeloma)

Waldenström macroglobulinemia (ORPHA 33226)

Primary systemic amyloidosis (ORPHA 314701)

POEMS syndrome (ORPHA 2905)

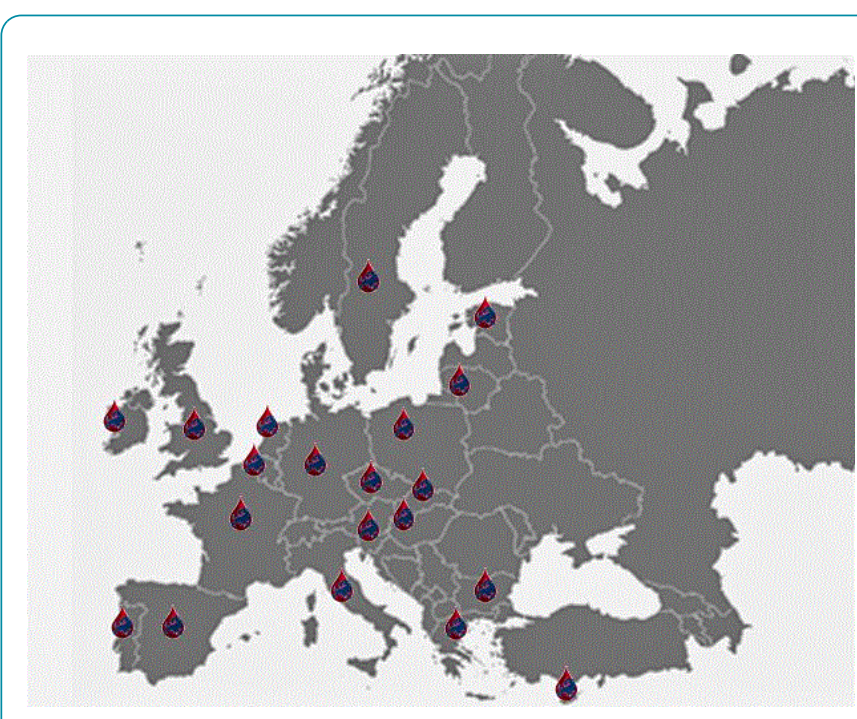

Figure 1: European countries who have expressed interest to pertain to EuroBloodNet.
Once established, the European Reference Network for Rare Haematological Diseases (EuroBloodNet) will include, at least 20 different European Countries (Figure 1) and will closely collaborate with other centers of expertise and networks at national and international levels. This will be a crucial step to improve the services for clinical management of both malignant and non-malignant rare haematological diseases, as well the education and patient's social care. Moreover, patients and health professionals will have an easy access to recognized centres of expertise for each rare haematological disease category and in turn, new opportunities to undertake innovative and useful actions based on the ERN.

\section{Acknowledgment}

We are indebted to ENERCA Consortium and to the European Haematology Association (EHA) for their coordinate efforts in the preparation and submission of the ERN in Rare Haematological Diseases (EuroBloodNet), and to the European Commission (EC) DG-SANCO and the Executive Agency for Consumers, Health, and Agriculture Food (CHAFEA) for cofinancing, from 2002 to 2016, the four phases of ENERCA Project.

\section{References}

1. Vives Corrons JL, Mañú Pereira MM (2015) Enerca: the european network for patients with rare anaemias. J Rare Dis Diagn Ther 1: 1-4

2. The European Parliament and the Council of the European Union (2011) Directive 2011/24/EU of the European Parliament and of the Council.

3. Enerca: Recommendations for Centres of Expertise in Rare Anaemias (2014) A white Book.

4. European Reference Networks Board of Member States Statement adopted by the ERN BoMS on affiliated partners (2016) European networks of reference for rare diseases.

5. Vives Corrons JL (2016) European reference network in hematology-the opportunity to boost hematology research, treatment and care. 